\title{
Turbulent Jet in a Rotating Fluid
}

\author{
By Hiroshi Niino* \\ Ocean Research Institute, University of Tokyo, Japan \\ (Manuscript received 19 May 1978, in revised form 25 October 1978)
}

\begin{abstract}
Properties of both a turbulent jet injected downward in a rotating fluid and the induced secondary circulation are investigated by means of laboratory experiments and a linear theory. The main results obtained by the laboratory experiments are summarized as follows:

1) The advancing velocity of the lower end of the jet is smaller than that in a static fluid.

2) A remarkable upward motion appears when the injection of the source fluid is stopped.

3) The secondary circulation induced by the jet does not penetrate far below the lower end.

The structure of the induced secondary circulation can be qualitatively explained by the linear theory in which a two-dimensional laminar jet is assumed.

The suppression of the vertical velocities is caused by the upward pressure gradient force which is generated by the entrainment of the rotating ambient fluid and is horizontally balanced with the centrifugal force acting on the swirling fluid particles as was considered by Barcilon (1967b) and Wilkins et al. (1971a). The existence of this pressure gradient force is consistent with 1) and 2). 3) indicates that the depression in the pressure near the nozzle cancels the high pressure near the lower end of the jet due to the divergence field so as to make the pressure perturbation far below the jet vanish.
\end{abstract}

\section{Introduction}

An important problem in tornadogenesis is to explain a process of vortex-stretching which produces an intense concentration of vorticity. This process may be triggered by a strong upward or downward motion of air mass in clouds. Concentrated release of latent heat may cause the upward motion. Localized cooling of the air mass due to evaporation of cloud and rain drops may cause the downward motion. In either case production of buoyancy must be maintained for an enough time to make an intense vortex, namely the tornado. The vertical air motion which produces vortex-stretching may take a form of a turbulent plume. Therefore, an investigation of the turbulent plume generated in clouds will be important to substantiate the above speculation about the process of vortex-stretching.

\footnotetext{
* Present affiliation: Department of Geosciences, Purdue University, Indiana.
}

General properties of turbulent plumes have been extensively studied related to convective processes near the ground and in cumuli. Similarity solutions have been obtained by Batchelor (1954) and Morton et al. (1956). However, these results can not be applied to the plume associated with tornadogenesis, because they are obtained only in a static fluid without vorticity. On the other hand, the plume generated in a mother cloud of tornado is expected to develop in ambient air with rather intense vorticity. The typical vorticity in the mother cloud is reported as $10^{-3} \mathrm{sec}^{-1}$ (Fujita and Grandoso, 1968) and even more intense vorticity can be expected in a region where a tornado is being generated.

There are several works that deal with turbulent plumes or thermals in a rotating fluid layer. In connection with the flow field in a dust devil, Barcilon (1967b) extended the work by Morton et al. (1956) to a rotating fluid and investigated difference of properties of a plume as a function 
of Rossby number Ro (the ratio of square root of vertical momentum flux to angular momentum) and Froude number Fo (square root of the ratio of kinetic energy flux to potential energy flux). However, he dealt with only flow fields in a steady state, so that it may be difficult to apply his results to a turbulent plume in a developing stage. Wilkins et al. (1969, 1971a and b) performed a series of studies to estimate the effects of rotation on both an individual thermal and successive thermals. They adopted the experimental situation in which a thermal or successive thermals are injected at the lower boundary of a rotating fluid and their behaviors were studied. Recently, Eskridge and Das (1976) reported a numerical experiment to simulate tornadogenesis based on an idea that a downdraft driven by the drag of precipitating particles triggers a tornado.

Motivation of the present study is to extend the work by Wilkins et al. to the following situation:

1) They considered successive thermals, but a plume is another possibility to generate a tornado and it is of interest to investigate effects of rotation on the plume.

2) They injected a thermal at the lower boundary. Thus, the Ekman boundary layer accompanying the secondary flow may interact with the plume. However, a plume which leads to the tornado is produced in a cloud, so we produce the plume within a fluid apart from the boundary.

3) We are also interested in the characteristics of a secondary circulation induced by the plume.

The rotation of the basic field is expected to modify characteristics of the plume through a secondary circulation induced outside the plume. The secondary circulation for a buoyant plume may be essentially the same as that for a nonbuoyant jet. Since we wish to investigate pure effects due to rotation, we only deal with nonbuoyant jets in this study.

In the next section the results of laboratory experiments are presented, where characteristics of jets and the secondary circulation are shown in a homogeneous rotating fluid. In section 3 the main structure of the secondary circulation is explained by making use of linear theory. The results are discussed in the last section.

\section{Laboratory experiment}

\subsection{Experimental apparatus}

Fig. 1 shows a sketch of the experimental apparatus used in the present study. A jet is

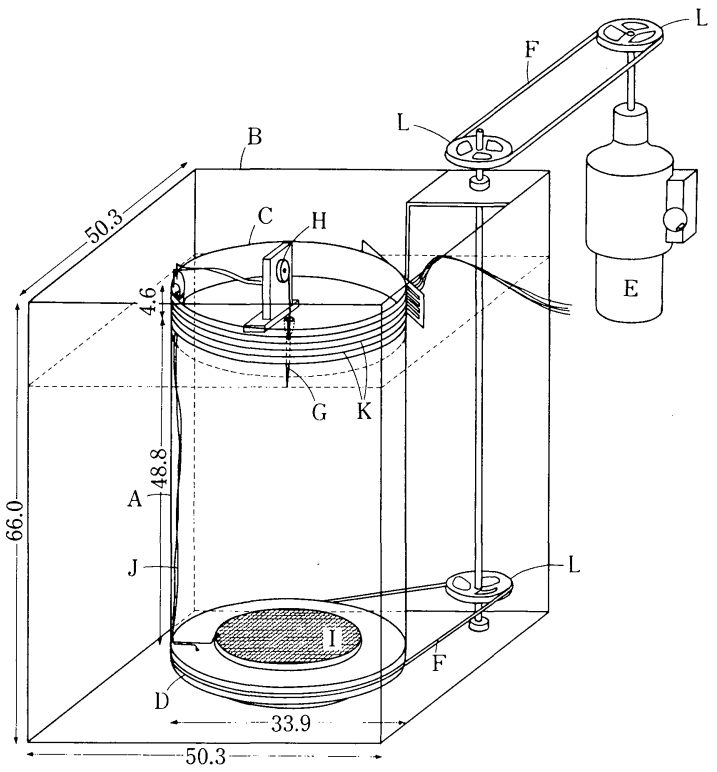

Fig. 1 A sketch of the experimental apparatus. The numerals in the figure show lengths in the unit of $1 \mathrm{~cm}$. A: $\operatorname{tank} A, B$ : $\operatorname{tank} B, C$ : tank C, D: turn-table, E: variable-speed motor, F: belts, G: nozzle, $\mathrm{H}$ : solenoid valve, I: copper net, J: electric lines, K: slip rings, L: pulleys.

injected in a cylindrical tank A made of Plexiglas which is filled with tap water. The depth of tank $A$ is $48.8 \mathrm{~cm}$ and the diameter is $33.9 \mathrm{~cm}$. It is submerged into a square tank B with plane vertical walls. The water is filled between these two tanks to eliminate distortion of images in tank A due to lens-effects of the cylindrical wall. A turn-table $D$ is afixed to the base of tank A. Turn-table $\mathbf{D}$ is driven by a variable-speed motor E (Simpo Kogyo, Ringcone, OM-100S) connected by belts $\mathrm{F}$ and pulleys $\mathrm{L}$. The accuracy of the rotation speed is unknown, but the rotation is so smooth that the irregularity of the rotation speed gives little effects on properties of jets. Source fluid for the jet (tap water) is kept in a cylindrical tank $\mathrm{C}$ with a diameter of $33.9 \mathrm{~cm}^{*}$ ) installed on tank A. A nozzle $G$ made of a glass tube is set at the center of the bottom of tank C. It is inserted into the working fluid. The outlet is fixed at $7.2 \mathrm{~cm}$ below the water surface. The volume flux of the jet is controlled by using nozzles with different diameters. There is a solenoid valve at the middle part of the nozzle

*) The diameter is $19.5 \mathrm{~cm}$ in Exp. 3 as discussed later. 
to initiate the injection at a given time*). In each experiment the depth of the source fluid in tank $\mathrm{C}$ is set to $4.0 \mathrm{~cm}$ initially and the source fluid is introduced into the working fluid in tank A by opening the valve. The duration is less than 20 seconds in each run. Change of the volume flux of the jet due to lowering of the depth of the source fluid is about $2 \%$ after 20 seconds. It is expected that the volume flux is also reduced when the source fluid is rotating. To avoid this effect we introduce the source fluid into tank $\mathrm{C}$ just before the injection**). Checks were made to confirm that the volume flux at the rotation speed of $1.3 \mathrm{rad} / \mathrm{sec}$ was the same as that for non-rotation, within an accuracy of $1 \%$ of the volume flux.

Procedures and results of 3 different experiments are described in the following sections. In each experiment injections of the jet are performed about 40 minutes after the time at which the turn-table is switched on. Forty minutes is long enough to complete the spin-up of the working fluid. ${ }^{* * *}$ )

The objectives of the experiments are as follows: Experiment 1 is to observe development of jets in a rotating fluid. Experiment 2 is to observe development of a secondary circulation in the ambient fluid caused by the injection of jets. Experiment 3 is to obtain a vertical profile of tangential velocities near a jet. Since experimental arrangements of these three experiments are rather different, it may be convenient to describe them separately.

\subsection{Experiment 1 (development of jets)}

(a) Experimental procedure

In this experiment flow visualization is performed with the $p H$-method: A small amount of thymol blue ( $p H$-indicator) is mixed into the working fluid. On the other hand, a dilute solu-

*) The injection was performed by a manual operation in Exp. 3 as stated later.

**) The time required for the source fluid to pass through the nozzle is about a tenth of the diffusion time assuming a laminar flow, so that there is a possibility that the source fluid injected into the working fluid is not adjusted to the rotation of the ambient fluid. However, this may have little effect on development of the turbulent jet, because the angular momentum of the source fluid is far smaller than that entrained from the ambient fluid.

***) The spin-up time of the fluid in tank $\mathrm{A}$ according to linear theory ranges from 7 to $10 \mathrm{~min}$ utes in our experiment. tion of $\mathrm{NaOH}$ colored by the thymol blue is used as the source fluid of the jet. When it is mixed with the working fluid, the color of the working fluid entrained into the jet becomes dark and the colored fluid, which is defined as the jet, can be clearly distinguished from the ambient fluid. The volume flux of the jet at the outlet of the nozzle in this experiment is $1.0 \mathrm{~cm}^{3} / \mathrm{sec}$ and $10.5 \mathrm{~cm}^{3}$ / sec (the diameter of the nozzle is $0.10 \mathrm{~cm}$ and $0.33 \mathrm{~cm}$, respectively).

In order to investigate flows near the bottom, a piece of copper net whose thickness is about $0.1 \mathrm{~mm}$ is set about $1 \mathrm{~cm}$ above the bottom (made by a brass plate) (cf. Fig. 1) and the fluid surrounding the copper net is colored by the $p H$ method (Baker, 1966) before the injection of the jet. If there are significant vertical motions, we can detect them from the displacement of the colored fluid. The experiments were performed for basic state rotation rates of $\Omega=0,0.7$ and $1.3 \mathrm{rad} / \mathrm{sec}$, respectively. When the volume flux of the source fluid $V$ is $1.0 \mathrm{~cm}^{3} / \mathrm{sec}$, observations were repeated 3 times for each rotation rate. When $V$ is $10.5 \mathrm{~cm}^{3} / \mathrm{sec}$, observations were repeated 4 times for $\Omega=0.0 \mathrm{rad} / \mathrm{sec}$, and 2 times for $\Omega=0.7 \mathrm{rad} / \mathrm{sec}$ and $\Omega=1.3 \mathrm{rad} / \mathrm{sec}$.

Development of the jet was recorded by a motor-driven Nikon F-2 camera with a $200 \mathrm{~mm}$ telescope lens and also by an $8 \mathrm{~mm}$ movie (Elmo 1018R). The jet was illuminated from the back by a scattered light. Although the start of the photographing is to be synchronized with opening of the solenoid valve of the nozzle, the first photograph is taken $0.2 \mathrm{sec}$ after the injection of the source fluid because of the mechanical time lag. Photographs are taken with the interval of $1.0 \mathrm{sec}$. The exposure time is $1 / 30 \mathrm{sec}$.

In order to determine objectively the positions of the leading edge of the jet from the photographs, a photo pattern analizer (Rhesca Co., Ltd., PPA-250) was used. It measures the brightness of a negative film and digitalizes it into 2048 grades. The lower end of the jet was defined as the position where there is no systematic horizontal gradient of the brightness. In a rotating fluid the photograph of the jet is sometimes disturbed by the electric lines as stated later and such a photograph is not used to determine the position.

\section{(b) Results}

An example of the development of a turbulent jet in a rotating fluid layer in comparison with that in a static fluid is shown in Fig. 2. The volume flux in both cases is $1.0 \mathrm{~cm}^{3} / \mathrm{sec}$. The 

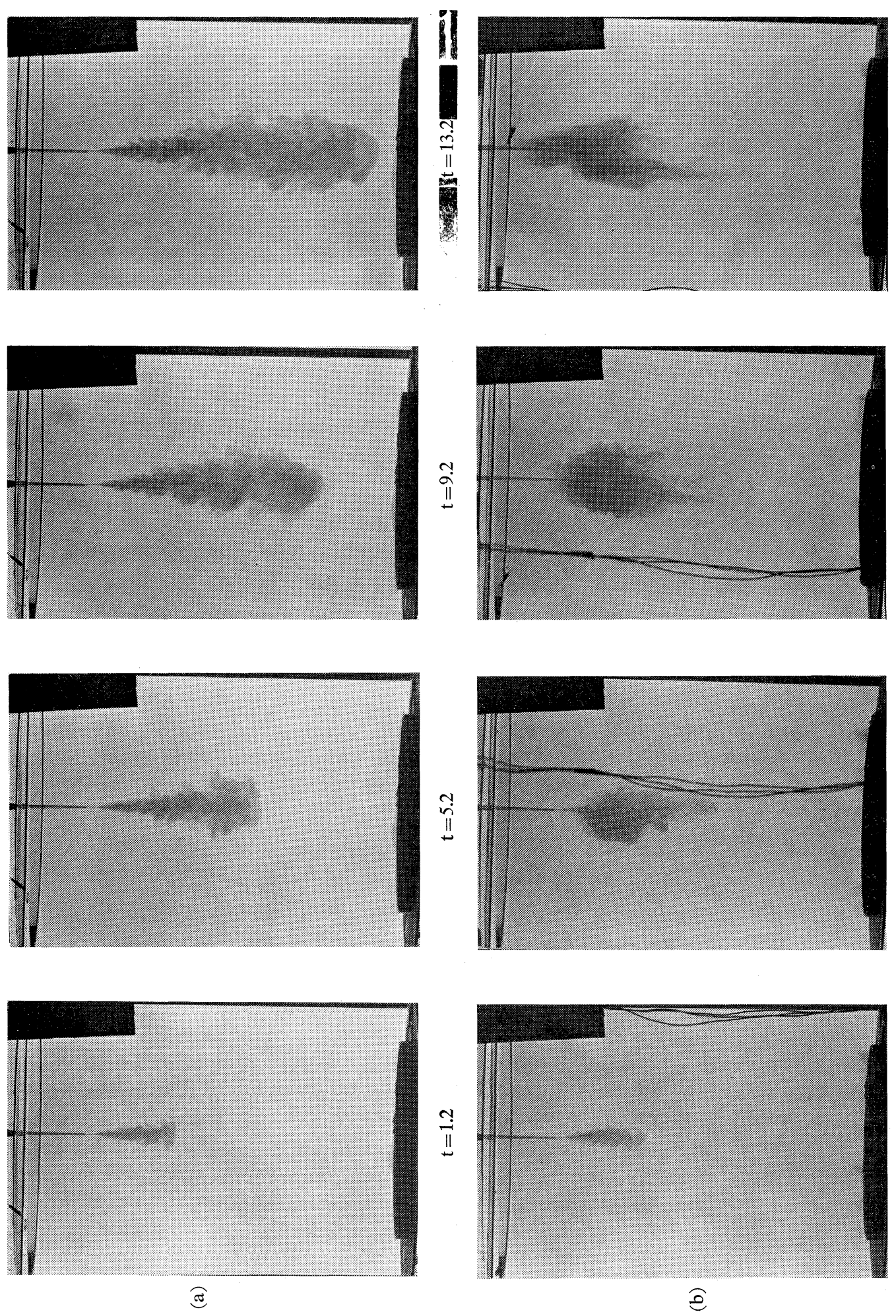

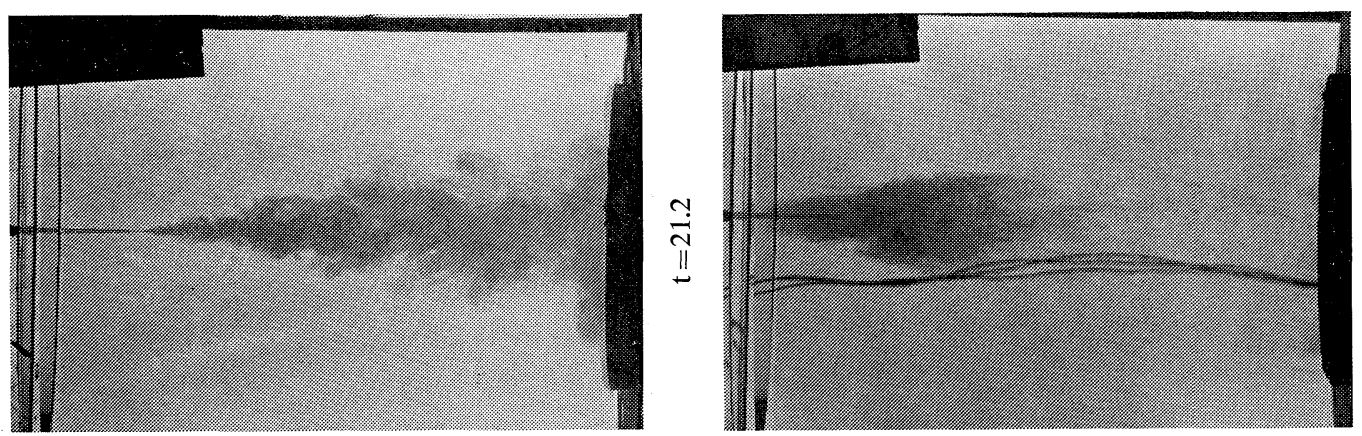

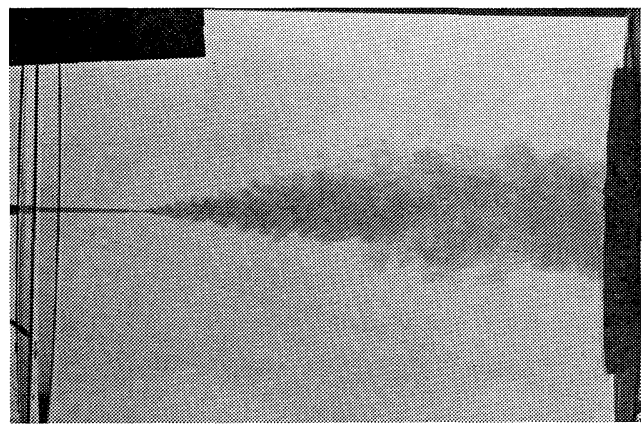

@

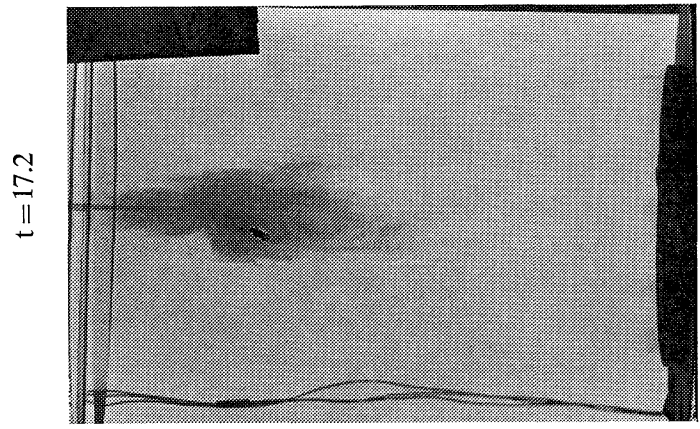

อ

Fig. 2 The development of the turbulent jet in a static fluid (a) and in a rotating fluid with $\Omega=1.3 \mathrm{rad} / \mathrm{sec}$ (b).

rotation rate of the basic state in Fig. 2(b) is 1.3 $\mathrm{rad} / \mathrm{sec}$. A circular frame at the bottom, which is seen as a dark thick line segment in the photographs, is a support of the copper net. A colored fluid is stored at the inner side of the frame. Thin and thick vertical lines seen in the photographs are wires to lift the electrodes and electric lines to give electric potential to the electrodes set at the bottom, respectively.

It is well-known that the jet in a static fluid develops in a conical shape. It is seen that fluid near the bottom is not affected by the jet until it comes to the bottom. It takes about 15 seconds for the jet to reach the bottom. The behavior of the jet in the rotating tank is remarkably different from that in the static fluid. The jet grows in the same manner as in the static fluid in the first few seconds, but the difference between the shapes of both the plumes become clear after 5.2 seconds (about one period of rotation). The diameter of the jet in a rotating fluid becomes small downward in the lower part in contrast with an inverted mushroom-like shape in the static fluid. The injected fluid stays just below the nozzle even after 9.2 seconds and the lower end is obscure compared with that in the static fluid. It should be noted that the injected fluid extends higher than the level of the outlet of the nozzle after 13.2 seconds. The colored fluid stored near the bottom shows no tendency to go upward throughout the whole stage of the development of the jet, implying that there is no significant upward motion that keeps the jet near the nozzle.

In Fig. 3 the position of the leading edge of the jet against the elapsed time after the initial injection of the source fluid is shown. The theoretical curves corresponding to those in a static fluid are also shown for comparison with the observed values. They are obtained as follows: The only external parameter given to the jet is the momentum flux $M$ at the nozzle or the modified momentum flux $Q=M / \pi=b^{2} w^{2}$ where $b$ is the radius of the nozzle and $w$ is the averaged vertical velocity at the outlet of the nozzle. The dimensional argument suggests that the position of the lower end of the jet $h$ is given by

$$
h=c \cdot Q^{1 / 4} \cdot t^{1 / 2},
$$

where $t$ is the time from the injection of the 


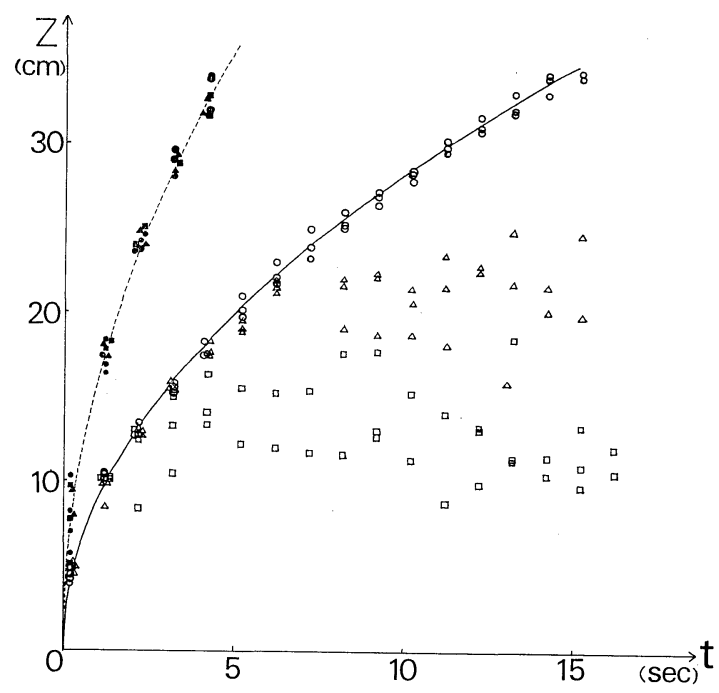

Fig. 3 The position of the lower end of the jet versus time from the injection of the jet both in a static fluid and in a rotating fluid. White and black marks show the observed positions when the volume fluxes of the source fluid $\mathrm{V}$ are $1.0 \mathrm{~cm}^{3} / \mathrm{sec}$ and $10.5 \mathrm{~cm}^{3} / \mathrm{sec}$, respectively. ( $\bigcirc \mathrm{O}: \Omega=0.0, \triangle \mathbf{\Delta}$ : $\Omega=0.7, \square \square: \Omega=1.3 \mathrm{rad} / \mathrm{sec})$. Solid and dashed lines show the theoretical curves obtained for $V=1.0$ and $10.5 \mathrm{~cm}^{3} / \mathrm{sec}$, respectively.

source fluid and $c$ is a constant which should be determined from experimental data (see Turner (1962)). The value of $c$ is found to be 3.51 . The theoretical curves are then obtained from equation (2.1) by substituting $c=3.51$ and $Q=$ $40.5 \mathrm{~cm}^{4} / \mathrm{sec}^{2}$ (for the volume flux of $1.0 \mathrm{~cm}^{3}$ / sec) or $Q=410 \mathrm{~cm}^{4} / \mathrm{sec}^{2}$ (for the volume flux of $\left.10.5 \mathrm{~cm}^{3} / \mathrm{sec}\right)$. It should be noted that the observed values in a static fluid agree fairly well with the theoretical values for the volume flux of both $1.0 \mathrm{~cm}^{3} / \mathrm{sec}$ and $10.5 \mathrm{~cm}^{3} / \mathrm{sec}$, respective1y. When the volume flux of the jet is large $(10.5$ $\mathrm{cm}^{3} / \mathrm{sec}$ ), the manner of the advance of the lower end in a rotating fluid does not seem to differ from that in a static fluid. On the other hand, when the volume flux of the jet is small $\left(1.0 \mathrm{~cm}^{3}\right.$ / sec), the behavior in a rotating fluid becomes remarkably different from that in a static fluid (except for a few seconds after the injection of the source fluid). The scatter of the calculated positions of the leading edge are rather large. However, one can observe a systematic tendency in spite of these fluctuations. When the rotation rate of the basic field is $0.7 \mathrm{rad} / \mathrm{sec}$, the positions fluctuate around $z=21 \mathrm{~cm}$, and when the rotation rate is $1.3 \mathrm{rad} / \mathrm{sec}$ the positions fluctuate around $z=13 \mathrm{~cm}$. It should be noted that the lower end of the jet stops at some level when the rotation rate is large enough (for a fixed value of $Q$ ) and that the distance from the nozzle to the level becomes smaller as the rotation rate becomes large.

\subsection{Experiment 2 (development of the secondary circulation)}

(a) Experimental procedure

In this experiment flow visualization is performed with aluminum flakes mixed into the working fluid. They are illuminated by a beam from a slide projector through a vertical slit of $1.5 \mathrm{~cm}$ width which is set at the side-wall of tank B. The illuminated region is a vertical cross section of tank A which contains the axis of that

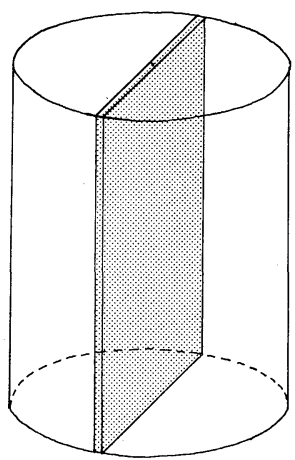

(a)

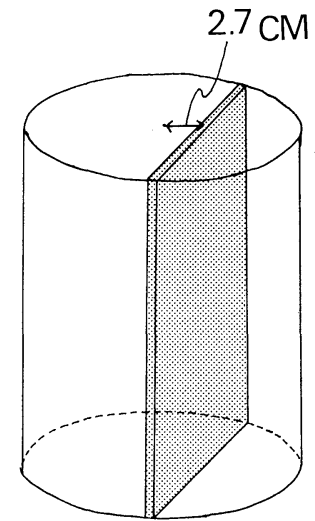

(b)

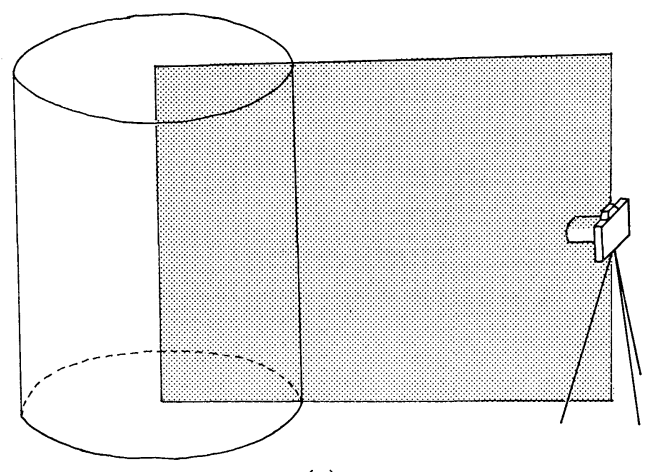

(c)

Fig. 4 Schematic figure of vertical planes to explain the observational system.

a) a vertical cross section which contains the axis of the tank A.

b) a vertical cross section the nearest distance of which from the axis of the tank $\mathrm{A}$ is $2.7 \mathrm{~cm}$.

c) a vertical plane that connects the axis of the tank A with the camera. 



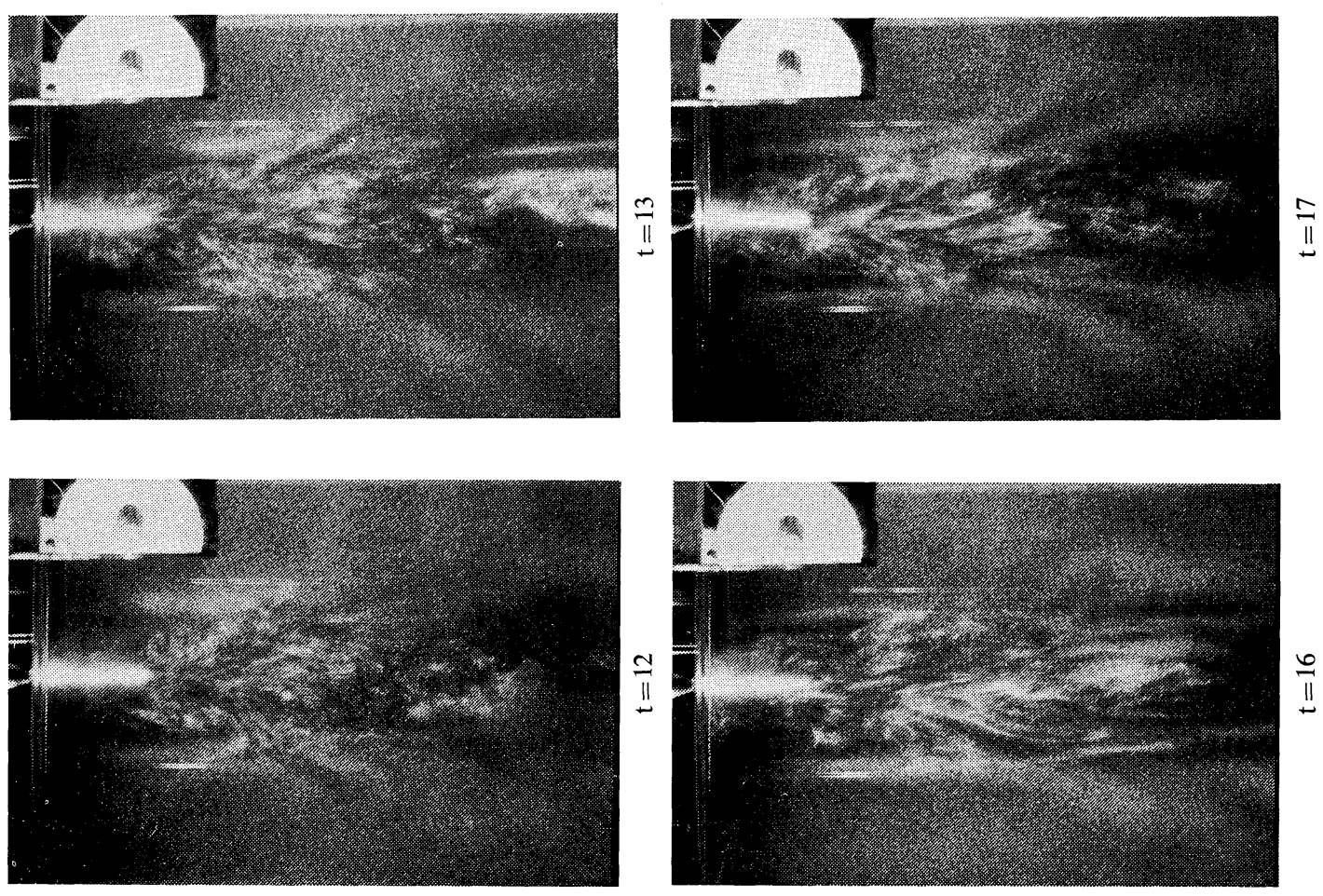

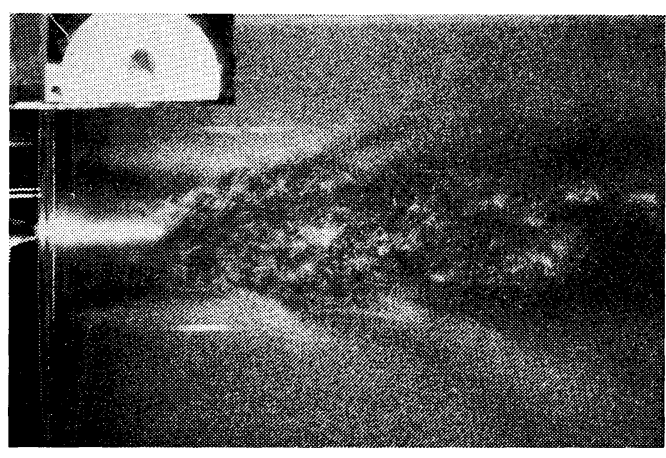

$\hat{e}$

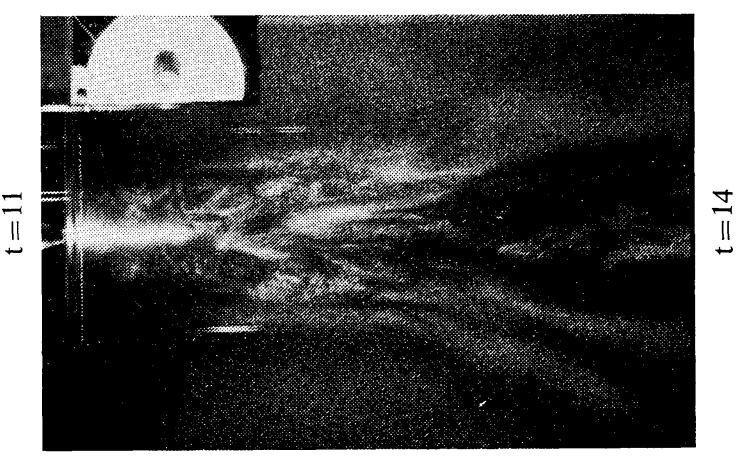

อ

Fig. 5 Time evolution of the jet and the secondary circulation both in a static fluid (a) and in a rotating fluid with $\Omega=1.3 \mathrm{rad} / \mathrm{sec}$ (b).

tank (see Fig. 4(a)). Since the flakes align in the region with a strong shear, this method is convenient to visualize eddies induced by the jet. Aluminum flakes are also mixed into the source fluid to observe flows within the jet. The volume flux of the jet at the outlet of the nozzle is fixed to $10.5 \mathrm{~cm}^{3} / \mathrm{sec}$ (the diameter of the nozzle is $0.33 \mathrm{~cm})$. The experiments are performed with a rotation rate of $1.3 \mathrm{rad} / \mathrm{sec}$, and one can compare the results with those in the static fluid. Development of the jet is photographed in the same manner as Experiment 1 except the expo- sure time is $0.5 \mathrm{sec}$.

\section{(b) Results}

Flows caused by the injection of the jet in a rotating tank are compared with those in a static fluid in Fig. 5. The manner of the development of the jet in a static fluid is the same as that in Experiment 1. It may be noted, however, that organized vortices are observed along the conical boundary of the jet. These eddies may be those that play a major role in the entrainment processes as pointed out by Townsend (1956). The first photograph in Fig. 5(b) shows the back- 
ground pattern before the injection of the jet. The dark and light lines seen near the rotation axis are considered to be caused by a small disturbance and should be regarded as a noise. After 3 seconds from the injection, a swirl is formed near the nozzle and it extends higher than the level of the outlet of the nozzle. After 6 seconds the shape of the jet resembles an ear of corn: the diameter of the jet increases more rapidly than that in the static fluid. It attains a maximum value at the middle level and then is reduced monotonously as observed in Experiment 1. The organized eddies which are seen in the static fluid are not clear. The diameter of the swirl seems to be close to the maximum diameter of the jet. It may be noted that the strong shear region of the swirl in the ambient fluid expands in the radial direction. This shear region seems to be the Stewartson layer which was demonstrated in the experiment of a bubble-driven tornado-like vortex by Turner (1966). The injection is stopped at 10 seconds. An upward motion then appears near the outlet of the nozzle, reaching the free surface of the working fluid. At the same time a disturbance produced by this upward motion propagates downward in the columnar form, implying the existence of columnar modes of inertial waves. Another feature seen in the photographs is conical lines radiating from the turbulent region, implying the existence of another mode of inertial waves, as well. Finally, the turbulent jet becomes a laminar two-dimensional swirl and disappears.

\subsection{Experiment 3 (the vertical profile of tangential velocity around a jet)}

(a) Experimental procedure

In this experiment flow visualization is performed with polystylene particles (the diameter is between 0.25 and $0.42 \mathrm{~mm}$ ) mixed into the working fluid. They are illuminated by a beam from a strobo-scope through a vertical slit of $1.0 \mathrm{~cm}$ width which is set at the side-wall of tank B. The illuminated region is a vertical cross section of tank A which is located at $2.7 \mathrm{~cm}$ from the axis of tank A (see Fig. 4(b)). Polystylene particles are also added into the source fluid. The volume flux of the jet at the outlet of the nozzle is fixed at $1.5 \mathrm{~cm}^{3} / \mathrm{sec}$ (the diameter of the nozzle is $0.21 \mathrm{~cm}$ ). The experiment is performed with a rotation rate of $0.7 \mathrm{rad} / \mathrm{sec}$. Photographs are taken in the direction normal to the illuminated region. Six flashings of the stroboscope with an interval of $0.15 \mathrm{sec}$ are recorded on the same film. The flashing duration is 20 microseconds. The photographing is repeated every $1.5 \mathrm{sec}$. In general, six dots are recorded on a trajectory of the tracer and we can obtain the velocity by measuring the distance between two dots further apart and by dividing it by the flashing interval. Calculations are carried out with tracers located near a vertical plane that connects the axis of tank A with the camera (see Fig. 4(c)).

\section{(b) Results}

Fig. 6 shows two vertical profiles of tangential velocities at $r=2.7 \mathrm{~cm}$ from the axis of tank A (which, strictly speaking, contain data between $r=2.2 \mathrm{~cm}$ and $r=3.2 \mathrm{~cm}$ because of the finite width of the slit). One is a profile for a background solid-body rotation where $\Omega=0.7 \mathrm{rad} /$ sec. The number of data points totals 36 , which are scattered around the average value of 1.86 $\mathrm{cm} / \mathrm{sec}$. This value represents the tangential velocity of the solid-body rotation at $r=2.7 \mathrm{~cm}$ for $\Omega=0.7 \mathrm{rad} / \mathrm{sec}$. The standard deviation is

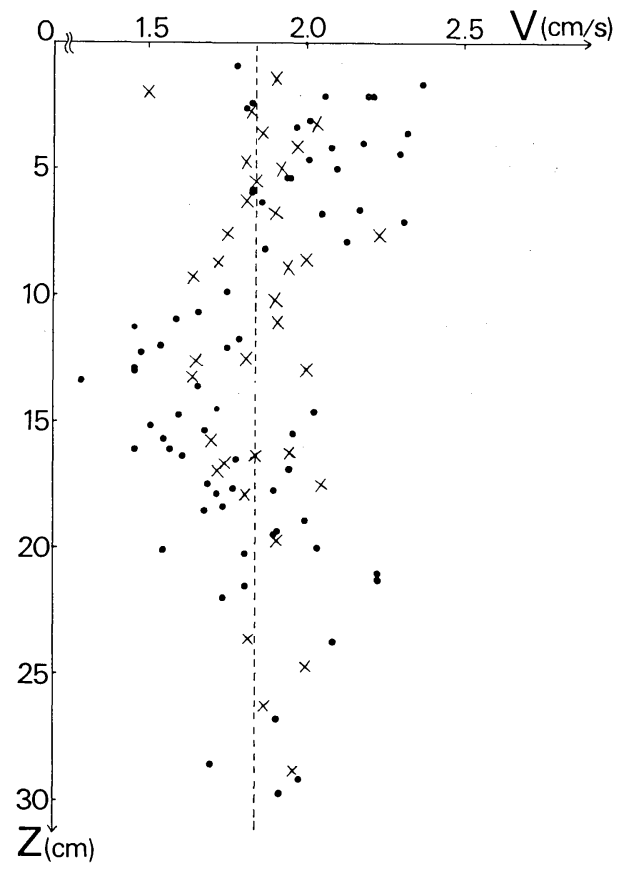

Fig. 6 Vertical profiles of tangential velocity at $r=2.7 \mathrm{~cm}$ from the axis of the tank A. The marks and $X$ show the tangential velocities obtained for a solid-body rotation $(\Omega=$ $0.7 \mathrm{rad} / \mathrm{sec}$ ) and for a state three seconds after the injection of the jet in that basic rotation, respectively. Dashed line shows the averaged velocity for the latter $(X)$. 
$0.14 \mathrm{~cm} / \mathrm{sec}$, and the data are distributed in the range of $1.86 \pm 0.37 \mathrm{~cm} / \mathrm{sec}$. The magnitude of the scatter also agrees well with that expected from the finite width of the slit. The other is a profile after three seconds from the injection of the jet in the basic state described above. The number of data points in this case is 73 . They are also scattered around an average value of $1.84 \mathrm{~cm} / \mathrm{sec}$, which is very close to that of the basic state. Comparing the manner of the scatter with that in the basic state, one can find that almost all the data above $z=9.0 \mathrm{~cm}$ (the vertical coordinate $z$ is measured downward from the outlet of the nozzle) are greater than average, but those between $z=9.0 \mathrm{~cm}$ and $z=18.0 \mathrm{~cm}$ are less than the average. The scatter of the data below $z=18.0 \mathrm{~cm}$ does not show any systematic tendency as well as that in the basic state. It is not likely that this manner of the scatter results from random errors of the data*). Rather this result suggests that the fluid outside the jet rotates faster than the basic state above $z=9.0$ $\mathrm{cm}$, and slower between $z=9.0 \mathrm{~cm}$ and $z=18.0$ $\mathrm{cm}$. The secondary circulation is nearly zero below $z=18.0 \mathrm{~cm}$. Unfortunately, the lowest position of the jet is not observed in this experiment. The position, however, can be estimated from the result of Experiment 1. It may be observed in Fig. 3 that the position of the lower end of the jet in a rotating fluid $(\Omega=0.7 \mathrm{rad} /$ sec) is almost the same as that in a static fluid after three seconds from the injection. Therefore one can estimate the position of the lower end of the jet from Eq. (2.1). Substituting the appropriate values $\left(Q=20.7 \mathrm{~cm}^{4} / \mathrm{s}^{2}, t=3 \mathrm{sec}\right)$, we get $h=13 \mathrm{~cm}$. Therefore, it may be plausible to consider that a secondary circulation induced

*) Consider the data in Fig. 6 divided into three groups: Group 1 consists of the data between $z=0.0 \mathrm{~cm}$ and $z=9.0 \mathrm{~cm}$; Group 2 between $z=$ $9.0 \mathrm{~cm}$ and $z=18.0 \mathrm{~cm}$; and Group 3 below $z=$ $18.0 \mathrm{~cm}$. For the data in Group 1 the average is $2.04 \mathrm{~cm} / \mathrm{sec}$ and the standard deviation is 0.17 $\mathrm{cm} / \mathrm{sec}$, while for those in Group 2 the average is $1.67 \mathrm{~cm} / \mathrm{sec}$ and the standard deviation is 0.17 $\mathrm{cm} / \mathrm{sec}$. For Group 3 the average is $1.91 \mathrm{~cm} / \mathrm{sec}$ and the standard deviation is $0.18 \mathrm{~cm} / \mathrm{sec}$. If one assumes that the population of each data group is subject to a Gaussian-distribution, it is easily proved with almost $100 \%$ probability that the averages of the population of Group 1 and Group 2 are greater and less than that for the basic state, respectively. The average of the population of Group 3, however, cannot be said to deviate from that in the basic state. by the jet does not penetrate far below the lower end of the jet. The main circulation outside the jet which consists of a cyclonic rotation in the upper half and an anti-cyclonic rotation in the lower half does not penetrate far below the jet.

\section{Linear theory}

In the previous section we observed that a turbulent jet in a rotating tank induces a swirl near the nozzle in the ambient fluid (cf. Exp. 2 and Exp. 3). Considering that the secondary flow is not observed near the bottom of the fluid tank, one can expect that the structure of the secondary circulation induced in the ambient fluid is able to be expressed as a transient response of an inviscid rotating fluid to the injection of the jet. If the secondary circulation is very weak, the characteristic Rossby number $(=\Delta \Omega / \Omega$, where $\Delta \Omega$ is the typical angular velocity of the perturbation and $\Omega$ is that in the basic state) is small. Then the induced circulation can be described by linearized equations of motion. It is of special interest to see whether the upward motion observed after the stop of the injection can be explained by the linear theory or not.

\subsection{Formulation of the problem}

Consider a fluid which is inviscid, incompressible and homogeneous, and a coordinate system as shown in Fig. 7 with the vertical $z^{*}$-axis. The fluid is rotating about the vertical axis with a constant angular velocity $\Omega$ and is bounded by a horizontal plate located at $z^{*}=0$. It is desirable to find a time dependent flow pattern caused by a given distribution of vertical velocity at the

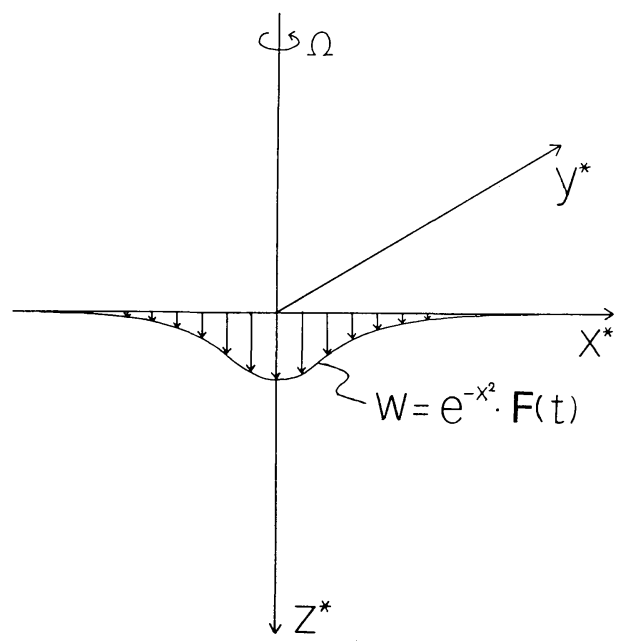

Fig. 7 A schematic figure of the coordinate system of the linear model. 
horizontal plate. Assume for simplicity that the distribution does not vary in the $y^{*}$-direction which is normal to the $x^{*} z^{*}$-plane, so that the response is uniform in the $y^{*}$-direction.

Let the characteristic velocity and horizontal scale of the forcing be $W$ and $D$, respectively. The non-dimensional variables are defined as:

$$
\begin{aligned}
& \left(\begin{array}{l}
x \\
z
\end{array}\right)=\frac{1}{D}\left(\begin{array}{c}
x^{*} \\
z^{*}
\end{array}\right), \quad\left(\begin{array}{c}
u \\
v \\
w
\end{array}\right)=\frac{1}{W}\left(\begin{array}{c}
u^{*} \\
v^{*} \\
w^{*}
\end{array}\right), \\
& t=f t^{*}, \quad \pi=\frac{1}{f D W} \pi^{*},
\end{aligned}
$$

where $u, v$ and $w$ are non-dimensional velocities in the $x$-, $y$ - and $z$-directions, respectively. $\pi^{*}$ is $p^{*} / \rho^{*}$, where $p^{*}$ is the pressure and $\rho^{*}$ is the density of the fluid. Time is scaled by the Coriolis parameter $f(=2 \Omega)$. The variables with an asterisk are the dimensional quantities. The governing equations in the non-dimensional form are written as

$$
\begin{aligned}
& \frac{\partial u}{\partial t}-v=-\frac{\partial \pi}{\partial x}, \\
& \frac{\partial v}{\partial t}+u=0, \\
& \frac{\partial w}{\partial t}=-\frac{\partial \pi}{\partial z}, \\
& \frac{\partial u}{\partial x}+\frac{\partial w}{\partial z}=0 .
\end{aligned}
$$

One can express the effect of the injection by the horizontal distribution of $w$ given by

$$
w=e^{-x^{2}} F(t)
$$

at $z=0$, where $F(t)$ describes the time variation of the forcing. (3.6) is different from the forcing in the laboratory experiment in the respect that the injection is given at the upper boundary in the linear model. As to the form of $F(t)$, we consider two functions:

$$
F(t)= \begin{cases}0 & \text { for }|t|>T \\ 1 & \text { for }|t| \leq T\end{cases}
$$

and

$$
F(t)=e^{-(t / T)^{2}},
$$

where $2 T$ is non-dimensional characteristic duration of the injection. The form of (3.7) gives the better approximation of the laboratory experiment, while the form of (3.8) is more convenient for numerical calculations than that of (3.7). Since the essential features of the flow patterns obtained with these two forms are similar as shown later, the main calculations are performed with (3.8). Let us find the solution of the governing equations which satisfies (3.6) at $z=0$ and

$$
w=0 \quad \text { at infinity. }
$$

\subsection{Calculation procedure}

At first let us try to find the solution which satisfies the boundary condition:

$$
w=e^{-i k x-i \omega t} \quad \text { at } z=0 .
$$

Eliminating $u, v$ and $\pi$ from the governing equations, one obtains

$$
\frac{\partial^{2}}{\partial t^{2}}\left(\frac{\partial^{2} w}{\partial x^{2}}+\frac{\partial^{2} w}{\partial z^{2}}\right)+\frac{\partial^{2} w}{\partial z^{2}}=0
$$

Assuming that $w$ is expressed as $w_{k, \omega}(z) \times$ $e^{-i k x-i \omega t}$, this equation becomes

$$
\left(1-\omega^{2}\right) \frac{\partial^{2} \omega_{k, \omega}}{\partial z^{2}}+\omega^{2} k^{2} w_{k, \omega}=0 .
$$

The solutions which satisfy (3.9) are

$$
\begin{aligned}
& w_{k, \omega}=\exp \left(-\frac{|\omega k|}{\sqrt{\omega^{2}-1}} z\right) \text { for }|\omega|>1 \\
& w_{k, \omega}=\exp \left(i \frac{\omega|k|}{\sqrt{1-\omega^{2}}} z\right) \text { for }|\omega|<1
\end{aligned}
$$

For $|\omega|=1$ there is no non-trivial solution which satisfies the boundary condition (3.9). The solutions (3.14) express inertial waves whose energy propagate downward.

Next we superpose the solutions for various values of $k$ and $\omega$ as

$$
\begin{aligned}
w(x, z, t)= & \frac{1}{4 \pi^{2}} \int_{-\infty}^{\infty} \int_{-\infty}^{\infty} a_{k, \omega} w_{k}, \omega \\
& \times e^{-\imath k x-i \omega t} d k d \omega,
\end{aligned}
$$

where $a_{k, \omega}$ is the weighting function.

If we put

$$
a_{k, \omega}=2 \sqrt{\pi} \exp \left(-\frac{k^{2}}{4}\right) \frac{\sin \omega T}{\omega},
$$

(3.15) satisfies the boundary condition (3.7). If we put

$$
a_{k, \omega}=T \pi \exp \left(-\frac{k^{2}}{4}\right) \exp \left(-\frac{T^{2} \omega^{2}}{4}\right),
$$

(3.15) satisfies the boundary condition (3.8). As $a_{k, \omega}$ is an even function of both $k$ and $\omega$, the range of integration in (3.15) can be replaced from $(-\infty, \infty)$ to $(0, \infty)$ after some modifications of the integrand. The numerical integration is performed by means of the trapezoid formula with $\Delta \omega=0.1$ and $\Delta k=0.1$. The upper limit of 

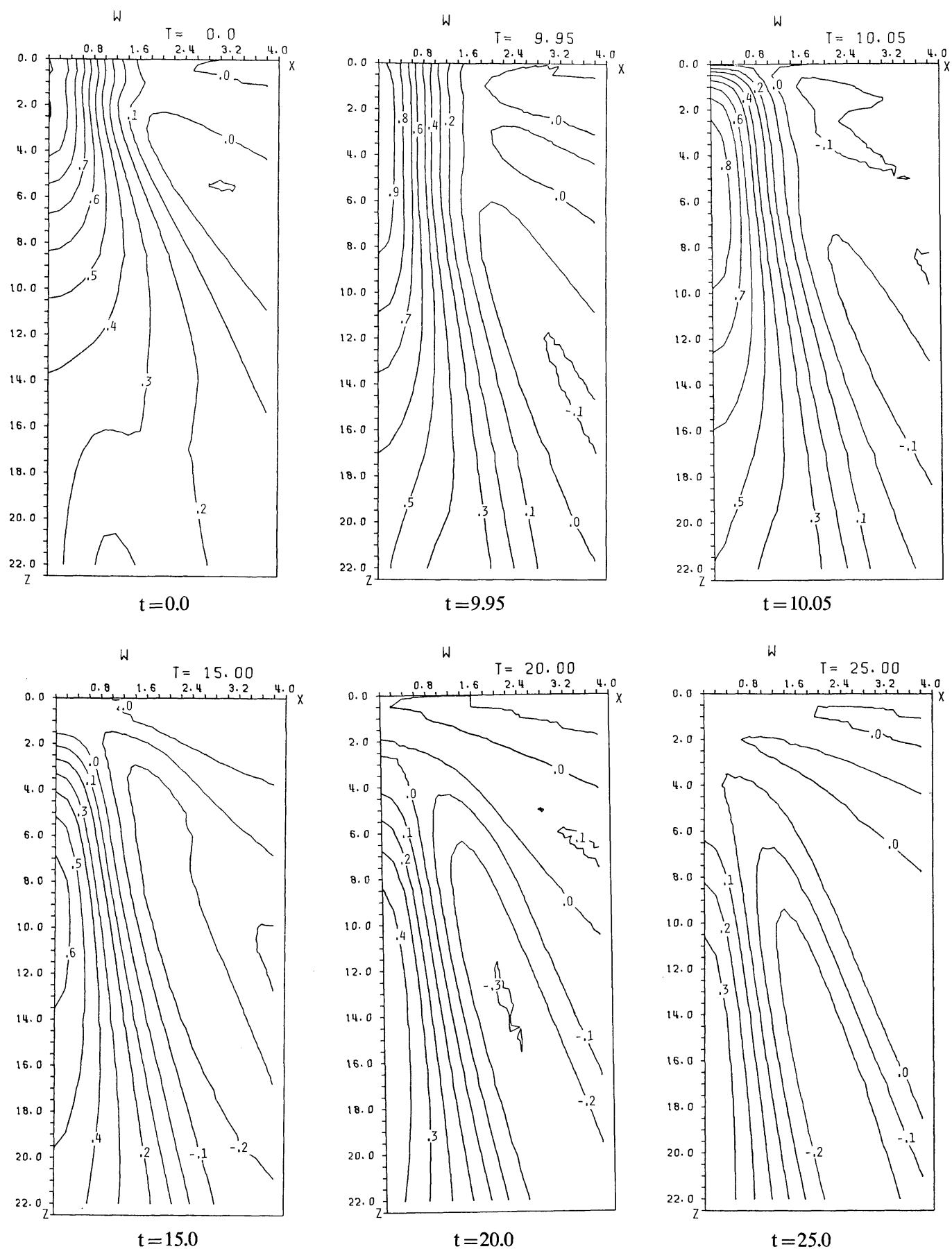

Fig. 8 Time evolution of the vertical velocity field produced by the forcing given by (3.7).

integrations is approximated by finite values; $k=$ is known. The value of the integrand at $\omega=1$ 6.0 and $\omega=5.0$ when the boundary condition is determined by the average of the integrand is given by (3.8) and $\omega=30.0$ when it is given at $\omega=1-\varepsilon$ and that at $\omega=1+\varepsilon . \varepsilon=10^{-3}$ is by (3.7). These upper limits give a relative error used throughout this calculation, after checking within $0.5 \%$ at $z=0$ where the exact integral that $\varepsilon$ in the range between $10^{-2}$ and $10^{-5}$ gives 

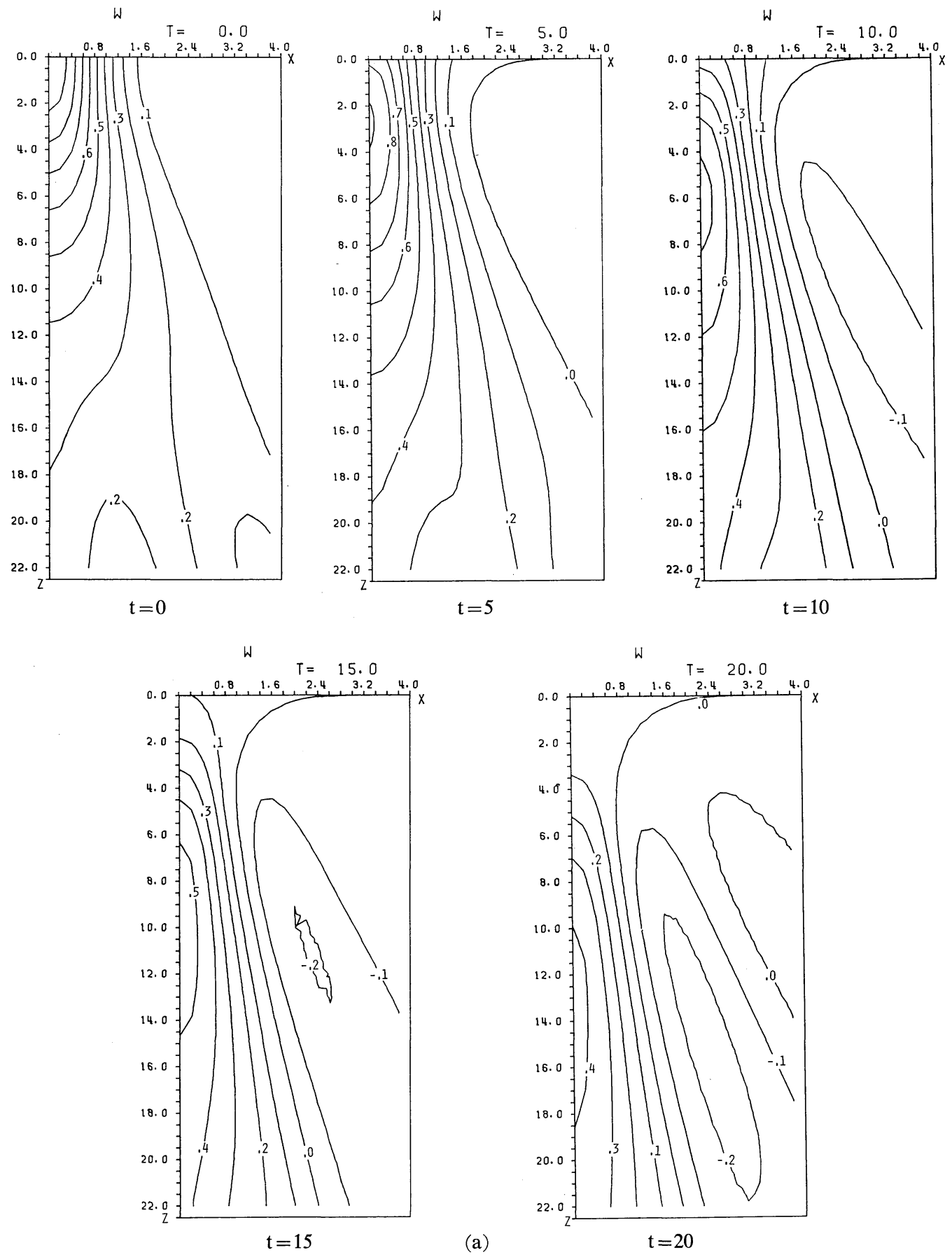

Fig. 9 Time evolution of the fields of the vertical velocity (a), the pressure (b) and the tangential velocity (c) produced by the forcing given by (3.8).

negligible differences. Pressure $\pi(x, z, t)$ and tangential velocity $v(x, z, t)$ are also obtained numerically in the same manner as the calculation of the vertical velocity $w(x, z, t)$.

3.3 Results
Figure 8 shows the results of the calculation of $w$ produced by the forcing given by (3.7). The injection is started at $t=-10$ and stopped at $t=10$. If the angular velocity of the fluid tank is $1.0 \mathrm{rad} / \mathrm{sec}$, the duration of the injection (20 

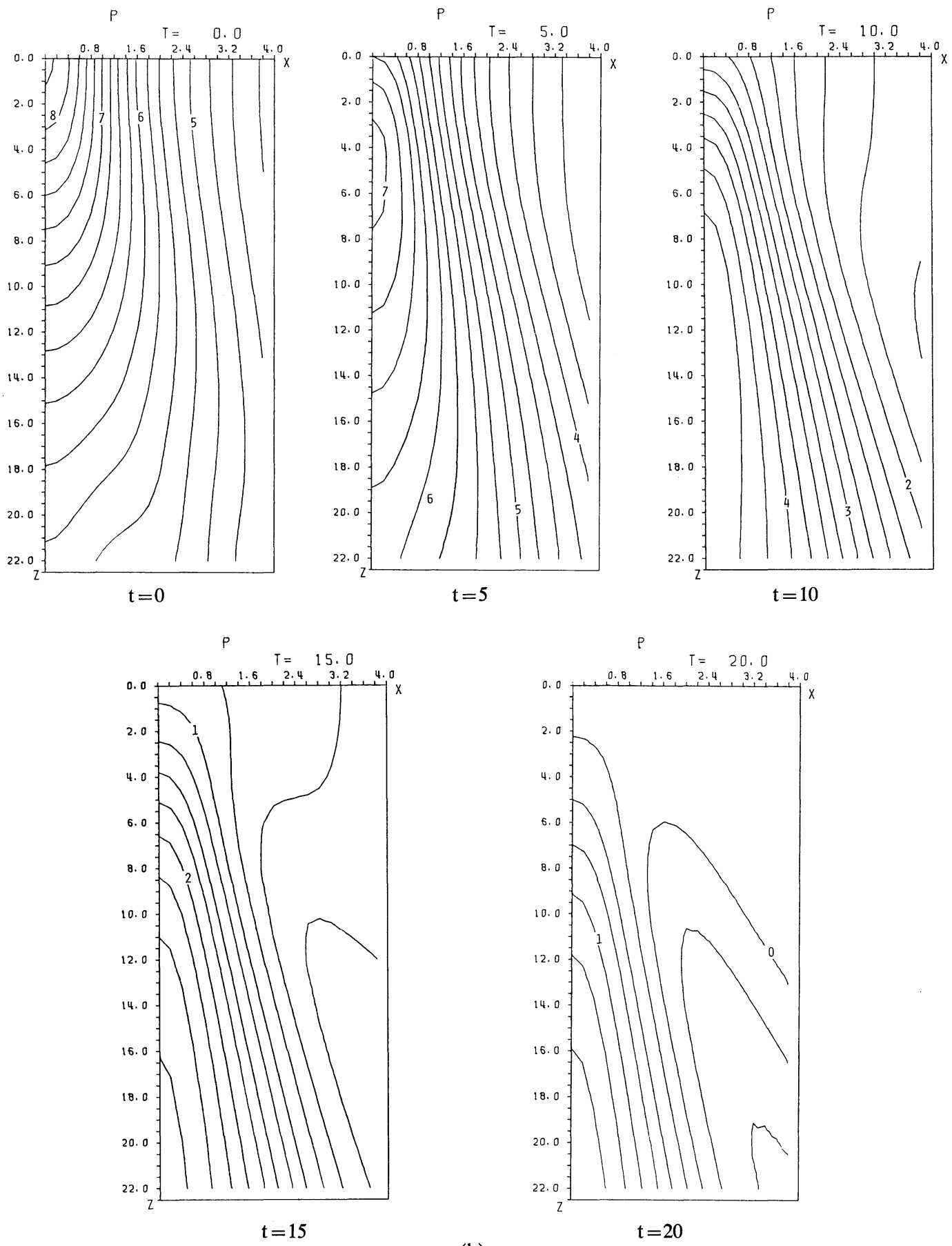

(b)

in non-dimensional time units) corresponds to 10 metric with respect to the $z$-axis, only the distrisec. The variation of $w$ from $t=10$ to $t=25$ is bution in the positive- $x$ region is shown. The shown in Fig. 8. Of interest are the flows induced forcing velocity is a maximum at the origin and outside the jet after the stop of the injection. it is reduced to $1 / \mathrm{e}$ at $x=1$. Since the downThe first figure shows $w$ at the middle period of ward flow near the vertical axis is laminar, it the injection. Since the pattern of $w$ is sym- does not correspond to the turbulent jet in the 

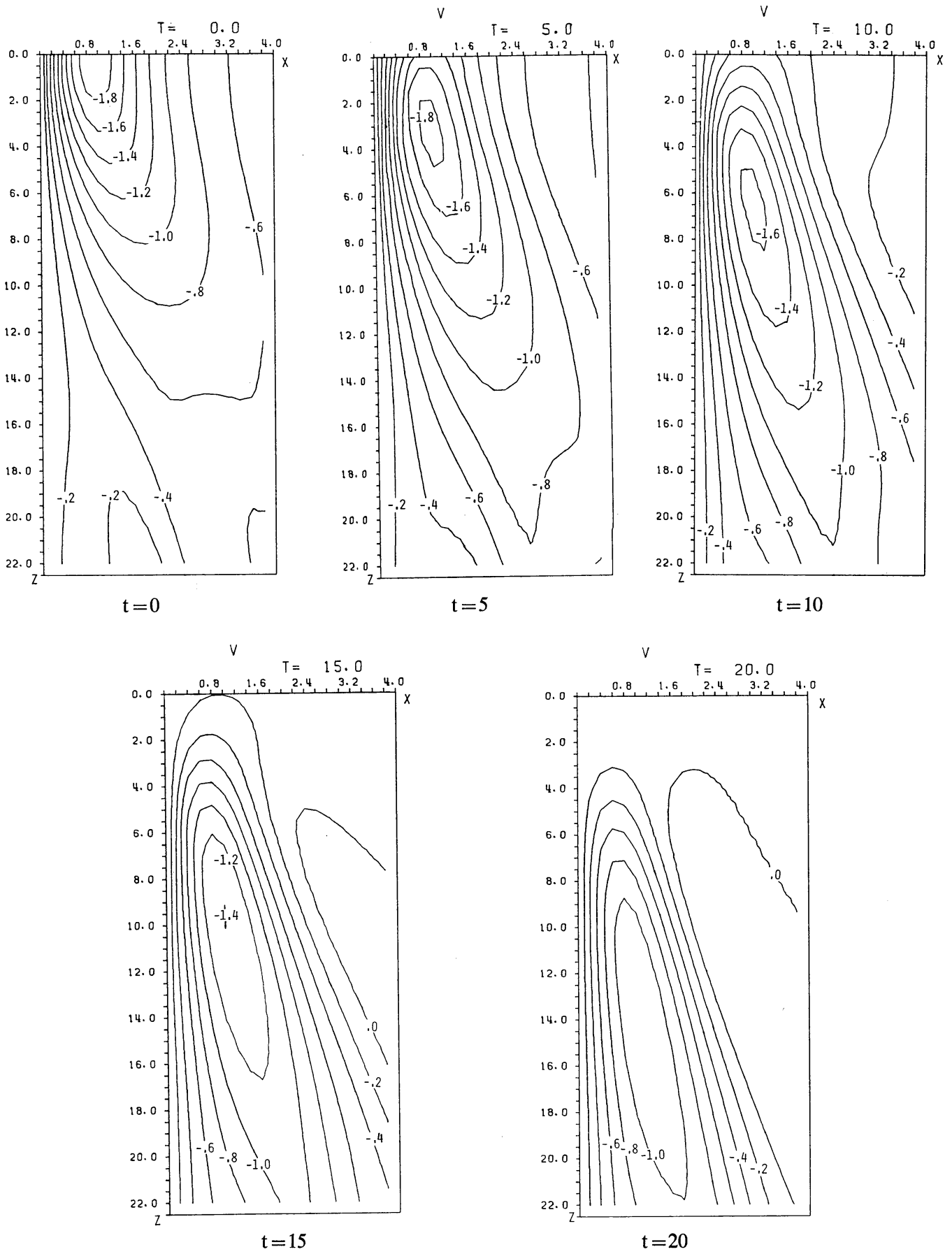

(c)

laboratory experiment. However, this will be at the time just before and after the stop of the called a "jet" in the following for convenience. injection. At $t=9.95$ the downward motion takes Our main interest is to observe flows outside this a columnar form in the upper portion and spreads downward motion. Notice that a very weak up- in the lower portion. The flow structure in this ward motion is seen even during the injection stage is similar to that in a developing Taylor of the jet. The next two figures show flows column. A linear analysis of the latter problem 
is given in Greenspan (1969). At $t=10.05$ upward motions appear just below the forcing level. The upward motion intensified and the area extends to the region surrounding the jet at $t=15.0$. The motion attains its maximum value near $t=$ 20.0 and decays after that.

Shown are the time evolution of $w, \pi$ and $v$ produced by the forcing given by (3.8) in Fig. 9(a), (b) and (c), respectively. The first figure of Fig. 9(a) is the distribution of $w$ at $t=0$, the time when the forcing becomes a maximum. Comparing this figure with the second figure of Fig. 8, it is noted that the distribution is almost the same except that the weak upward motion does not appear and that the columnar pattern of the jet is less remarkable. The time evolution of $w$ is then quite similar to that of Fig. 8. Therefore the time evolution of $v$ and $\pi$ are calculated only for the forcing given by (3.8), considering the economy of the time of the computation.

At $t=0$ the pressure field is characterized by vertical isobars whose pattern is similar to that of the vertical velocity (the first figure of the Fig. 9(b)). The pressure is high in and near the jet which diverges downward. At $t=5$ the position of the highest pressure shifts to about $z=5$ and the isobars deform from the columnar pattern to inclined lines, implying that there prevails upward pressure gradients outside the jet. At $t=10$ the inclination of the isobars becomes more remarkable. After that the evolution of the patterns of the pressure field is nearly similar to that of the vertical velocity. On the other hand, the pattern of the tangential velocity at $t=0$ (the first figure of Fig. 9(c)) suggests that it is nearly in a geostrophic balance. The velocity is anticyclonic*) and the maximum value is observed in the region where the horizontal pressure gradient is largest. At $t=15$ a region appears where the tangential velocity is cyclonic outside the jet, corresponding to a striking deformation of the pressure field. After that the cyclonic region extends to the upper level. On the other hand, the main part of the anti-cyclonic circulation moves downward, accompanied by the downward development of the jet.

\section{Discussion of the results}

The main characteristics of a turbulent jet in-

*) Here, anti-cyclonic means that a fluid particle rotates slower than the rotating frame. It does not correspond to the negative vorticity in our co-ordinate system. jected downward in a rotating fluid may be summarized as follows:

1) The advancing velocity of the lower end of the jet is smaller than that of a jet in a static fluid.

2) A strong upward motion appears in and around the jet when the injection of the source fluid is stopped. If the rotation rate of the basic state exceeds a certain value, the upward motion appears around the jet even before the stop of the injection.

3) The secondary circulation induced by the jet does not penetrate far below the lower end of the jet, implying that the influence of the bottom of the fluid tank is not significant.

Discussion of each of these characteristics is given in the following subsections.

\subsection{Suppression of vertical velocities}

Suppression of the advancing velocity of a thermal or successive thermals in a rotating fluid has been reported by Wilkins et al. (1969, 1971a). Although a jet is used instead of thermals in our experiment, the mechanisms of the suppression may be similar to each other.

Wilkins et al. (1971a) considered that rotation of the ambient fluid gives two kinds of effects to a thermal or successive thermals: One effect is to make a swirl outside the jet, because the outside fluid is entrained into the jet conserving the angular momentum. Then a centrifugal force acting on a swirling fluid particle prevents the ambient fluid from going inward and eventually a balance between the centrifugal force and the horizontal pressure gradient force is attained. On the other hand, the swirl is strongest near the nozzle where the entrainment is maximum. Then, the pressure depression near the nozzle may be greater than that in the lower part. Downward motion of the jet is expected to be suppressed by the vertical pressure gradient force thus produced**). Another effect is to suppress a horizontal mixing by establishing cyclostrophic balance. The latter effect does not suppress the vertical velocity.

Our experimental results are consistent with their speculation in the following points:

1) According to the result of Experiment 2, a swirl is formed near the nozzle and it extends to the level higher than that of the outlet of the nozzle 3 seconds after the start of the injection. This fact suggests that the cyclostrophic balance

**) This effect was qualitatively predicted by Barcilon (1967b). 
prevails near the nozzle.

2) According to the results of Experiment 3 (Fig. 6), the tangential velocity outside the upper part of the jet shows a cyclonic deviation from the basic state, while outside the lower part an anti-cyclonic rotation is observed. This implies that there is a vertical pressure gradient in the jet which has a tendency to suppress the vertical velocity.

3) Although there is a possibility that the swirl induced by the jet propagates downward as low frequency inertial waves and reaches the bottom, producing an upward current through the Ekman pumping associated with the Ekman boundary layer formed at the bottom of the fluid tank, the results of Experiment 1 suggest that this mechanism has little significance to the suppression of the vertical velocity (cf. section 4.3).

4) The organized eddies observed in a static fluid are not apparent in a rotating fluid. This may suggest that the turbulence in the jet and the mechanism of the entrainment are modified by the effect of the rotation field. Townsend (1956) suggested that the indentations due to the large eddies make the entrainment effective. In a rotating fluid, however, it is expected that the large eddies are easily modified by the rotation and, in fact, the above result suggests that the entrainment may be less effective than that in a static fluid. Then, the initial vertical velocity is maintained longer than a jet in a static fluid. In our experiment, however, the vertical velocity is suppressed by the rotation. This result suggests that the effect of the vertical pressure gradient seems to play a more dominant role than the modification of the entrainment in determining the vertical velocity of the jet.

As shown in Fig. 3, the lower end of the jet stopped at a certain level in a rotating fluid. This is an extreme manifestation of the suppression of the advancing velocity. It is of interest to study how the penetration depth of the jet depends on the parameters given to the jet. To get a qualitative idea, let us consider a simple mechanical model, and a cylindrical coordinate as shown in Fig. 10. An inviscid, homogeneous and incompressible fluid is confined in a region between $z=-d$ and $z=0$. At the bottom of the region a cylinder with a piston with the radius $R$ is connected. The whole system rotates with the angular velocity $\Omega$ around the $z$-axis. The downward motion of the piston induces an inward current in the fluid layer between $z=0$ and $z=-d$ and the fluid near $r=R$ rotates faster

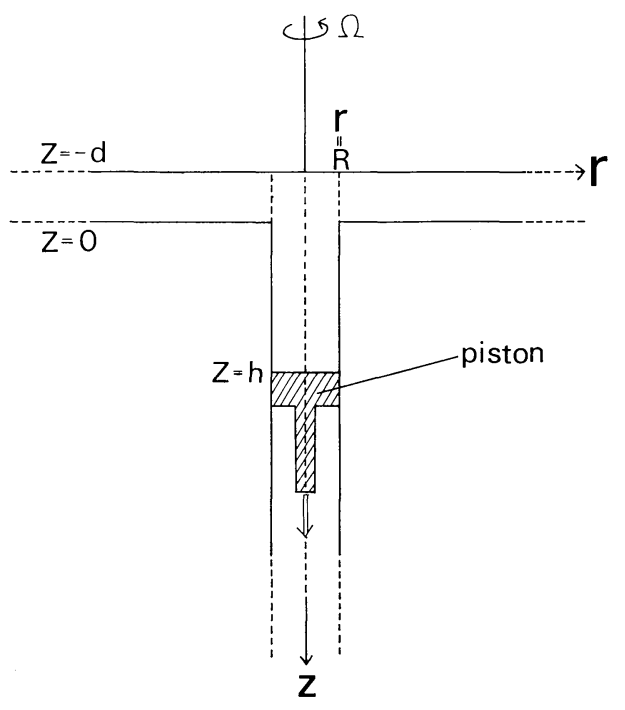

Fig. 10 A schematic of the mechanical model.

than that in the basic field due to the conservation of the angular momentum. Then the pressure above the piston decreases. If we neglect the gravity, the amount of the pressure depression $\overline{p^{*}}$ is given by

$$
\overline{p^{*}}=-\frac{3 \rho \Omega^{2} D^{2}}{4} h^{2},
$$

where $\rho$ is the density of the fluid, $h$ is the travelling distance of the piston and $D$ is defined by $R / d$ (see Appendix). Let us consider that the pressure difference between the lower end of the plume located at $z=h$ and the outlet of the nozzle is given by (4.1).

When the vertical pressure gradient is present, the momentum conservation for the jet is given by

$$
\frac{d}{d z}\left(\pi b^{2} w^{2}\right)=-\frac{d}{d z}\left(\pi b^{2} \cdot \frac{p}{\rho}\right),
$$

where $b$ is the radius of the plume, $w$ is the vertical velocity in the jet, $p$ is the pressure and $\rho$ is the density of the fluid. Integrating this equation, we get

$$
b^{2} w^{2}+b^{2} \cdot \frac{p}{\rho}=\text { const. }
$$

At the level where the lower end of the jet stops, $w=0$ and $p=-\overline{p^{*}}$, if we allow $p=0$ at the outlet of the nozzle. Then, (4.3) becomes

$$
-b^{2} \overline{p^{*}}=Q,
$$

where $Q$ is the modified momentum flux. Then, 
putting $b \sim R$ for simplicity and substituting (4.1) is into (4.4), one obtains

$$
h=\frac{2}{\sqrt{3} D R} \cdot \frac{\sqrt{Q}}{\Omega} .
$$

This gives the maximum penetration depth. Since $D=R / d$ and $R$ is uncertain for the jet, one can put

$$
h=c^{\prime} \cdot \frac{\sqrt{Q}}{\Omega}
$$

and leave $c^{\prime}$ as a constant which should be determined from laboratory experiments. From Fig. $3, h=21 \mathrm{~cm}$ for $Q=40.5 \mathrm{~cm}^{4} / \mathrm{sec}^{2}$ and $\Omega=$ $0.7 \mathrm{rad} / \mathrm{sec}$, and $h=13 \mathrm{~cm}$ for the same $Q$ and $\Omega=1.3 \mathrm{rad} / \mathrm{sec} . \quad c^{\prime}$ is found to be $2.3 \sim 2.7$. If $c^{\prime}=2.5$, we have $h=39 \mathrm{~cm}$ for $Q=410 \mathrm{~cm}^{4} / \mathrm{sec}^{2}$ and $\Omega=1.3 \mathrm{rad} / \mathrm{sec}$. This may explain the reason why the lower end of the jet does not stop at some level when $Q=410 \mathrm{~cm}^{4} / \mathrm{sec}^{2}$ in Fig. 3, because the distance between the bottom of the tank A and the outlet of the nozzle is $36 \mathrm{~cm}$ in our experiment.

\subsection{Upward motion}

We have shown using the linear theory that weak upward motions appear and develop outside the jet just after the stop of the forcing in Fig. 8 and 9. The behavior is qualitatively similar to that observed in the laboratory experiment. However, we can expect development of a stronger upward motion from the results of the linear theory in Section 3. Let us consider that a fluid exists also over the horizontal boundary at which (3.6) is given as the boundary condition of the upper fluid. The physical meaning of the forcing in this system is to push the fluid at $z=0$ downward by some mechanism like a pump located at $z=0$. From the consideration of the symmetry of the solution about $z=0$, we find that the flow structure above the forcing is essentially the same as that below the forcing. For the fluid below the forcing $(z>0)$ the $z$-derivative of the vertical velocity is negative before the stop of the injection as shown in Fig. 8 and 9. Therefore $\partial u / \partial x$ is positive from the mass continuity, meaning the divergence. The pressure is high and the tangential velocity is anti-cyclonic. Symmetry of the solution leads to a result that for $z<0$ the vertical velocity and its $z$-derivative are positive, meaning the convergence in the $x-y$ plane. The pressure is low and the tangential velocity is cyclonic. This means that the pressure and the tangential velocity are discontinuous at $z=0$. Discontinuity of the tangential velocity is possible in the inviscid fluid. However, the pressure must be continuous at $z=0$, if there is no rigid boundary at $z=0$. In fact, a vertical pressure gradient generated between just above and below the forcing by the secondary flow is sustained by the forcing $\mathfrak{F}$ confined at $z=0$ such that

$$
\frac{\partial w}{\partial t}=-\frac{\partial \pi}{\partial z}+\Im
$$

$\mathfrak{F}$ can be regarded as a pump with infinitesimal thickness which pushes the fluid downward against the pressure gradient induced by the secondary flow. From the equations (3.2), (3.3), (4.7) and (3.5), one can write,

$$
\nabla^{2} \pi=\frac{\partial v}{\partial x}+\frac{\partial \widetilde{z}}{\partial z} .
$$

When the forcing is stopped, (4.8) becomes

$$
\nabla^{2} \pi=\frac{\partial v}{\partial x}
$$

This equation says that the pressure perturbation vanishes everywhere in a static fluid when the injection is stopped. In a rotating fluid, $\partial v / \partial x$ remains after discontinuing the forcing and an upward motion is produced by the resulting pressure gradient $\partial \pi / \partial z$ across the forcing level. However, this upward motion is not expressed in our linear theory developed in section 3 , because $w$ at the forcing level is externally given as a boundary condition.

Although the jet in the laboratory experiment is turbulent and the non-linearity is predominant

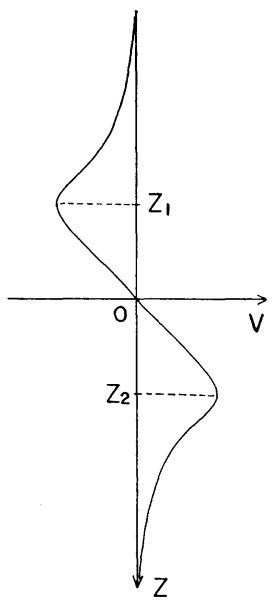

(a)

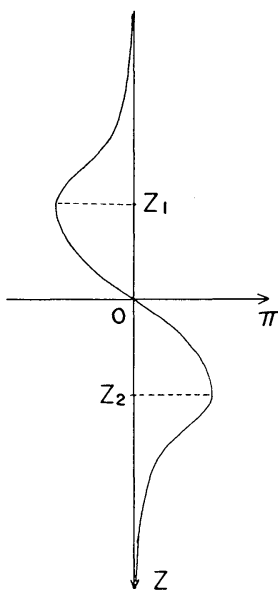

(b)
Fig. 11 Schematic vertical profiles of the tangential velocity (a) and the pressure (b) expected from the linear model. 
in the jet, the essential features of the secondary circulation around the jet may be explained by the linear theory as follows: The most important non-linear term is the self-advection term $w$ $(\partial w / \partial z)$ in the vertical momentum equation. It should be noted that the term $w(\partial w / \partial z)$ expresses the effects of a pump which sucks the ambient fluid into the jet and pushes it downward. We can regard this term as a forcing $\mathfrak{F}$ appearing in the equation (4.7). Now $\mathfrak{F}$ distributes in the $z$-direction in the laboratory system, but the effect can be expressed by superposing the solutions for the forcing given by the function as considered in the linear theory. Thus extending the results of the linear theory mentioned above, one can understand the qualitative picture of our experiment. Figure 11(a) shows a schematic vertical profile of the tangential velocity near the axis of the tank in the laboratory system before the injection is stopped, which is expected from the linear theory. The tangential velocity is cyclonic near the outlet of the nozzle $\left(z=z_{1}\right)$ and anti-cyclonic near the lower end of the jet $\left(z=z_{2}\right)$. This speculation is also supported from Fig. 6. The corresponding pressure field may have a distribution as shown in Fig. 11(b). If the pressure gradient between $z=z_{1}$ and $z=z_{2}$ is sufficiently large, the upward motion may be produced when the injection is stopped.

The upward motions appearing in Fig. 8 and 9 are considered to be produced essentially by the same mechanism described above.

4.3 Influence of the lower boundary of the fluid tank

It is an unexpected result that the bottom of the fluid tank has little influence on characteristics of the jet in a rotating fluid layer. Although the injection of the source fluid induces a cyclonic rotation near the nozzle, an anti-cyclonic rotation is also induced in the lower level so that pressure perturbations at the lower boundary (which is far below the lower end of the plume) vanish. The high pressure due to the divergence at the leading edge of the jet is expected to be nearly equal to the low pressure due to the cyclonic swirl. This picture is qualitatively obtained by the measurement of the tangential velocity (see Fig. 6). However, as stated in section 2.3 (b) there certainly exist inertial waves forced by the jet. A part of the energy of the jet must be transported to the bottom in the form of the inertial waves. The emission of the inertial waves by a turbulent jet in a rotating fluid is of interest but outside the scope of the present study.

\section{Acknowledgements}

The present work is the revised form of the author's M.S. thesis submitted to Geophysical Institute, University of Tokyo. The author would like to express his hearty thanks to Prof. T. Matsuno for valuable advice as a thesis supervisor. $\mathrm{He}$ is grateful to Prof. R. Kimura, Ocean Research Institute, University of Tokyo, for sincere guidance, many valuable suggestions and encouragements throughout this work. $\mathrm{He}$ is also much indebted to Prof. T. Asai, Ocean Research Institute, University of Tokyo and Prof. E. M. Agee, Purdue University, for useful comments on improving the paper. Thanks are extended to Messrs. N. Misawa and K. Ishikawa for the excellent design and construction of the experimental apparatus and Miss C. Okubo, Miss V. Ewing and Mrs. G. Shelley for typewriting the manuscript.

\section{Appendix}

It is assumed that the fluid particles between $z=0$ and $z=-d$ for $r>R$ can move only in the horizontal plane. Dropping the friction terms, the equations of motion in the inertial frame become

$$
\begin{aligned}
& -\frac{v^{2}}{r}=-\frac{1}{\rho} \cdot \frac{\partial p}{\partial r}, \\
& \frac{\partial(v r)}{\partial t}+u \frac{\partial(v r)}{\partial r}=0,
\end{aligned}
$$

where $u$ and $v$ are the velocity components in the radial and tangential direction, respectively. The term $d u / d t$ in the first equation is neglected for simplicity (quasi-cyclostrophic balance is assumed). The conservation of mass gives for $r>R$

$$
u(r, t)=\frac{u(R, t) R}{r},
$$

where $u(R, t)$ is the radial velocity at $r=R$.

Then the position of the piston is predicted as

$$
\frac{d h}{d t}=-\frac{2 d}{R} u(R, t) .
$$

If we put $U(t)=u(R, t) \cdot R$ and $V(r, t)=v \cdot r$, (A.2) becomes

$$
\frac{\partial V}{\partial t}+\frac{U}{r} \cdot \frac{\partial V}{\partial r}=0
$$

after substituting (A.3). Then putting $x=r^{2} / 2$

$$
\begin{gathered}
\text { and } \tau=\int_{0}^{t} U d t, \text { (A. 5) becomes } \\
\frac{\partial V}{\partial \tau}+\frac{\partial V}{\partial x}=0
\end{gathered}
$$


General solution for (A.6) is $V(x, \tau)=f(\tau-x)$ where $f(y)$ is an arbitrary function of $y$, and therefore

$$
V(r, t)=f\left(\int_{0}^{t} U d t-\frac{r^{2}}{2}\right)
$$

At $t=0$, the fluid is rotating as a solid-body, that is, $V=\Omega r^{2}$, so

$$
f\left(-\frac{r^{2}}{2}\right)=\Omega r^{2} \text {. }
$$

Therefore $f(y)=-2 \Omega y$ and one can obtain

$$
v(r, t)=\Omega\left(r-\frac{2 R}{r} \int_{0}^{t} u(R, t) d t\right) .
$$

Considering that $\int_{0}^{t} u(R, t) d t=-\frac{R}{2 d} h$ from (A.4),

(A.7) yields

$$
v(r, t)=\Omega\left(r+\frac{R D h}{r}\right), \quad(r>R)
$$

where $D$ is defined by $R / d$. Hereafter we consider the domain; $\sqrt{h / d} \gg r / R>1$ so that the basic rotation rate is small in comparison with the induced rotation rate. Let us calculate the pressure $p^{*}$ viewed from the rotating frame. Above assumption assures us to neglect the Coriolis force term. The corresponding equation to (A.1) in the rotating frame is

$$
-\frac{v^{* 2}}{r}=-\frac{1}{\rho} \frac{\partial p^{*}}{\partial r},
$$

where * denotes a quantity viewed from the rotating frame and

$$
v^{*}=v-\Omega r=\frac{R \Omega D h}{r} \text {. }
$$

using (A.8). Integrating (A.9) from $r=\infty^{*}$ ) to $r=R$ and putting $p^{*}=0$ at $r=\infty$,

$$
\left[\frac{p^{*}}{\rho}\right]_{r=R}=-\frac{1}{2} \Omega^{2} D^{2} h^{2} .
$$

In order to calculate the pressure $p^{*}$ for $r<R$, it is assumed tentatively that the fluid is in a solid-body rotation for $r<R$. As $v^{*}(r=R)=\Omega D h$ from (A.10), $v^{*}(r)$ is assumed that

*) Since $\sqrt{h / d \gg r / R}$ should be satisfied, the meaning of $r=\infty$ is asymptotic.

$$
v^{*}(r)=\frac{v^{*}(r=R)}{R} r=\frac{\Omega D h}{R} r .
$$

Then, from (A.9)

$$
\begin{aligned}
& {\left[\frac{p^{*}}{\rho}\right]_{r=r}=\left[\frac{p^{*}}{\rho}\right]_{r=R}-\frac{\Omega^{2} D^{2} h^{2}}{2 R^{2}}\left(R^{2}-r^{2}\right),} \\
& \frac{p^{*}}{\rho}=\frac{1}{\pi R^{2}} \int_{0}^{R} \frac{p^{*}}{p} \cdot 2 \pi r d r=-\frac{3 \Omega^{2} D^{2} h^{2}}{4} .
\end{aligned}
$$

This result is (4.1).

\section{References}

Barcilon, A., 1967b: A theoretical and experimental model for a dust devil. J. Atmos. Sci., 24, 453466.

Baker, D. J., 1966: A technique for the precise measurement of small fluid velocities. J. Fluid Mech., 26, 573-575.

Batchelor, G. K., 1954: Heat convection and buoyancy effects in fluids. Quart. J. Roy. Meteor. Soc., 80, 339-358.

Eskridge, R. and P. Das, 1976: Effect of a precipitation-driven downdraft on a rotating wind field: a possible trigger mechanism for tornadoes? J. Atmos. Sci., 33, 70-84.

Fujita, T. T. and H. Grandoso, 1968: Split of a thunderstorm into anticyclonic and cyclonic storms and their motions as determined from numerical model experiments. J. Atmos. Sci., 25, $416-439$.

Greenspan, H.P., 1969: The theory of rotating fluids. Cambridge University Press, $328 \mathrm{pp}$.

Morton, B. R., G. I. Taylor and J. S. Turner, 1956: Turbulent gravitational convection from maintained and instantaneous sources. Proc. Roy. Soc. London, A, 234, 1-23.

Townsend, A. A., 1956: The structure of turbulent shear flow. Cambridge University Press, $429 \mathrm{pp}$.

Turner, J. S., 1962: The 'starting plume' in neutral surroundings. J. Fluid Mech., 13, 356-368.

Wilkins, E. M., Y. Sasaki, E. M. Friday, J. McCarthy and J. R. McIntyre, 1969: Properties of simulated thermals in a rotating fluid. J. Geophys. Res., 74, $4472-4486$. cessive thermals. Mon. Wea. Rev, 99, 215-226.

$\longrightarrow$, and $\longrightarrow, 1971 b$ : Vortex formation by successive thermals: a numerical simulation. Mon. Wea. Rev., 99, 577-592. 


\title{
回転流体中の乱流ジェット
}

\author{
新野宏 \\ 東京大学海洋研究所
}

回転流体中において鉛直下向さに注入された乱流ジェットの性質及びそのジェットによってひき怙こされる二次流 れの性質を室内実験と線形論により調べた。室内実験によって得られた主な結果をまとめると次のようになる。

1）回転流体中に拈いては, ジェットの先端の進む速度は静止流体中のそれに較べて小さい。

2）回転流体中では，ジェットのもととなる流体の注入を停止したときに顕著な上昇流が生ずる。

3）ジェットによってひき拉こされる二次流れはジェットの先端より十分下のレベルまでは及ばない。

ジェットによってひきおこされる二次流れの構造は, 二次元の層流ジェットを仮定した線形論によって定性的に説 明することが可能である。

鉛直速度の抑制は，Barcilon (1969b) 及び Wilkins et al. (1971a)によって考えられたよらに，回転している流 体粒子に㗢く遠心力と水平方向につりあった気圧傾度力がジェットの進向方向と逆向き（上向き）の成分を持つ為に おこる。この上向きの気圧傾度力の存在は 1)・2）の結果をうまく説明する。3）の結果はノズル付近の収束場に伴う 低圧がジェットの先端付近の発散場に伴ら高圧とほとんど打消し合い, ジェットの十分下での圧力の摂動をほとんど 0にしていることを示唆している。 